\title{
Singlet Exciton Fission in Solution
}

Brian J. Walker ${ }^{1}$, Andrew J. Musser ${ }^{1}$, David Beljonne ${ }^{2} \&$ Richard H. Friend ${ }^{1 *}$

1_ Cavendish Laboratory, University of Cambridge

J. J. Thomson Avenue, Cambridge, United Kingdom

${ }^{2}$ - Laboratory for Chemistry of Novel Materials, Université de Mons

Place du Parc 20, 7000 Mons, Belgium

Singlet exciton fission, the spin-conserving process that produces two spin triplet excited states from one photoexcited singlet state, is a means to circumvent the ShockleyQueisser limit in single-junction solar cells. While the process through which singlet fission occurs is not well-characterised, some local order is thought to be necessary for intermolecular coupling. Here, we report $200 \%$ triplet yield and triplet formation rates approaching the diffusion limit in solutions of TIPS-pentacene. We observe a transient bound excimer intermediate, formed by collision of one photoexcited, and one ground state TIPS-pentacene molecule. The intermediate breaks up when the two triplets have separated to each TIPS-pentacene molecule. This efficient system is a model for future singlet fission materials and for disordered device components that produce cascades of excited states from sunlight.

First proposed to account for the optical and magnetic properties of crystalline anthracene $^{1}$ and tetracene $e^{2,3}$, singlet exciton fission ${ }^{4,5}$ is the reverse process of triplet-triplet annihilation $^{6}$. In singlet exciton fission, a singlet excited state decays to form two triplet excitons on neighbouring molecules. Because the two product triplets have a net spin of zero, the singlet fission process conserves spin and is thus a faster means to generate triplets than 
intersystem crossing for organic chromophores ${ }^{7}$. When the fission process is exergonic ${ }^{8}$ this process can be rapid and efficient, as is now established for solid films of pentacene $\mathrm{e}^{9,10}$. Singlet fission has gained interest as a means to circumvent the Shockley-Queisser limit in solar cells ${ }^{11}$. Because singlet fission converts high-energy photons into two excitons, a singlet fission sensitiser can be used with a low band gap solar cell to reduce losses due to relaxation. For such a single-junction cell the maximum theoretical efficiency increases to $44 \%{ }^{12}$, and this potential has led to recent realisations of singlet fission-sensitised solar cells ${ }^{13,14}$.

Some basic aspects of the singlet fission mechanism remain unresolved. Although the energetic requirements for singlet fission are satisfied in a range of materials, experiments have shown that this is not the only criterion for efficient singlet fission ${ }^{15}$. While the nature of singlet fission suggests that the process requires exciton coupling ${ }^{16-21}$, the observation of fission in amorphous solids ${ }^{22}$ indicates that long-range order is not required. Singlet fission occurs for tetracene in both single crystals ${ }^{7}$ and polycrystalline films ${ }^{23}$, but the triplet population is also controlled by processes such as diffusion and annihilation that complicate analysis $^{24}$. While molecular solids are practical for devices, they give little insight into the short-range molecular interactions that govern singlet fission.

To address these questions, we investigated the molecular mechanism of singlet fission in solutions of TIPS-pentacene (Fig. 1a). Originally developed for use in field effect transistors $^{25}$, TIPS-pentacene (Fig. 1b) has two triisopropylsilylethynyl (TIPS) groups that lead to favourable intermolecular orientations and high hole mobilities in the solid state ${ }^{26}$. The substituents also render TIPS-pentacene far more soluble than unsubstituted pentacene ${ }^{27}$, allowing our solutions to reach concentrations of $3.1 \%$ by mass $(0.075 \mathrm{~mol} / \mathrm{L})$ in chloroform before saturation. 


\section{$\underline{\text { Results and Discussion }}$}

The absorption spectrum of dilute TIPS-pentacene solution is shown in Fig. 1c. For comparison, we also took the absorption spectrum of a solid-state film of TIPS-pentacene. Whether the film is produced through spin or drop-casting, the solid-state absorption spectrum has a red-shifted aggregate feature that is not present in solution. We took absorption spectra of solutions across several orders of magnitude in TIPS-pentacene concentration (Supplementary Fig. S1), and we observed no significant aggregates even in samples near the saturation point. We conclude that TIPS-pentacene molecules in solution are electronically decoupled in the ground state, even at high concentration. In a control experiment, we investigated the TIPS-pentacene solutions at different concentrations using diffusion-ordered nuclear magnetic resonance spectroscopy (DOSY). These spectra show that the diffusion constant of TIPS-pentacene $\left(9.7 \times 10^{-10} \mathrm{~m}^{2} / \mathrm{s}\right)$ is unchanged across the series, indicating that the motion of TIPS-pentacene molecules is identical for concentrations from $10^{-5} \mathrm{~mol} / \mathrm{L}$ to $0.075 \mathrm{~mol} / \mathrm{L}$ (Supplementary Fig. S2).

\section{Quantitative singlet fission yield}

We probed the excited state dynamics of TIPS-pentacene solutions using transient absorption spectroscopy. This pump-probe technique is a popular means of studying photophysics, due to its versatile time resolution and its ease of comparison with ground-state absorption spectra $^{28}$. In transient absorption, a short pulse of light at a well-defined energy

(the "pump") excites the sample, and after a time delay ranging from femtoseconds to hundreds of microseconds, a pulse broad in energy but short in time (the "probe") interrogates the same spot. The transmitted light from the probe is compared with and without the pump light, and resolved by both spectral wavelength and delay time. If there is a 
change in the spectrum of the probe due to bleaching of the ground state transitions ("ground state bleach"), stimulated emission, or excited-state absorption from one excited state to another, then these will manifest as a change in the transmittance of the probe, $\Delta \mathrm{T}$. We report the signal normalised by the ground state transmittance, $\Delta \mathrm{T} / \mathrm{T}$, to facilitate comparison across experimental configurations.

We show a transient absorption spectrum for a dilute TIPS-pentacene solution $\left(10^{-4} \mathrm{~mol} / \mathrm{L}\right)$ in Fig. 2a. The electronic delay enables measurements from $10^{-9}-10^{-3} \mathrm{~s}$, although the initial signals are broadened by the temporal distribution of photons in the excitation pulse. Across the spectral range from 0.77 to $2.39 \mathrm{eV}$, the dynamics are dominated by features with a lifetime of $13 \mathrm{~ns}$. Specifically, we observe a ground state bleach at $1.9 \mathrm{eV}$, analogous to the $0-0$ band of the ground state absorption spectrum. There are also various excited state absorptions due to transitions to higher lying excited states (see Supplementary Figs. S3 and S4 for TA data above $2.3 \mathrm{eV}$ ). A spectral cross section at the peak of the signal intensity (2.6 ns) is shown in Fig. $2 \mathrm{~b}$, and because the lifetime correlates with the singlet lifetime measured during time-resolved photoluminescence spectroscopy (see below) it is identified as the TA spectrum of singlets, ${ }^{1} \mathrm{TP} *$.

The transient absorption spectrum of concentrated TIPS-pentacene solution $(0.075 \mathrm{~mol} / \mathrm{L})$ is shown in Fig. $2 \mathrm{c}$. In contrast to the absorption spectrum of ground state TIPS-pentacene (TP), there are major differences between TA spectra at low and high concentration of TIPS-pentacene. In the concentrated solution, there are negative features that correspond to excited-state absorptions. As these intense features have identically long lifetimes $(6.5 \mu \mathrm{s})$, they are distinct from the singlets in Fig. 2c and are correlated with one another. The concentrated solutions also have an intense, long-lived excited state absorption feature at $2.44 \mathrm{eV}$ (Supplementary Figs. S3 and S4), which has been assigned to the excited state absorption of triplets ${ }^{29}$. To confirm this assignment, we measured a dilute solution of 
TIPS-pentacene with the triplet sensitiser $N$-methylfulleropyrrolidine ${ }^{30}$, and the sensitiser and TIPS-pentacene spectra were deconvoluted using singular value decomposition. The resulting TIPS-pentacene spectrum, dominated by triplets, is shown in Fig. $2 b$ together with the singlet spectrum described above. Notably, the triplet control spectrum has the three infrared features that match the concentrated TIPS-pentacene solution. As a result, we infer that the long-lived species in concentrated TIPS-pentacene solution is the triplet, ${ }^{3} \mathrm{TP}^{*}$.

Representative kinetic traces from concentrated TIPS-pentacene are shown in Fig. 3a. As in Fig. 2a the kinetics are convoluted with the temporal breadth of the excitation pulse, and during this pulse the dynamics of ${ }^{1} \mathrm{TP} *$ and ${ }^{3} \mathrm{TP} *$ resemble each other. The subsequent decay of ${ }^{1} \mathrm{TP}^{*}$ is matched by further rise in ${ }^{3} \mathrm{TP} *$ until the singlets have fully decayed at 11 ns. From 10 ns onward, the triplet does not rise further. The ${ }^{3} \mathrm{TP}^{*}$ excited state absorption is mirrored in the ground state bleach (Fig. S5), whose intensity approximately doubles in the interval from $2.6 \mathrm{~ns}$ to $10 \mathrm{~ns}$. Because the ground state bleach arises from molecules in excited states, its continued increase demonstrates that additional TIPS-pentacene molecules are excited after the pump pulse has passed. Moreover, the bleach signals decay with a single-exponential lifetime of $6.5 \mu$ s, which closely resembles the ${ }^{3} \mathrm{TP}^{*}$ dynamics identified above. This correspondence indicates that after $10 \mathrm{~ns}$ the only excitations that remain on TIPS-pentacene are triplets. Together these observations suggest that efficient singlet fission occurs in concentrated TIPS-pentacene solutions. We attribute the delayed appearance of the feature at $1.65 \mathrm{eV}$ to the presence of stimulated emission, and as this decays the underlying triplet becomes apparent.

Because of the isotropic nature of TIPS-pentacene solutions and the absence of interferences in the infrared, we are able to determine the triplet yield (see Supplementary Section 4 for details). The key to this calculation is the relationship between singlet and triplet excited state absorption coefficients. For this isotropic solution, photoexcited TIPS- 
pentacene molecules have the same measured ground state bleach whether they are ${ }^{1} \mathrm{TP}^{*}$ or ${ }^{3} \mathrm{TP}^{*}$. Conveniently, the ground state bleach feature associated with either triplets or singlets in the range 1.9-2.0 eV is well separated from other spectral responses, as seen in Fig. 2b. We can therefore normalise the ${ }^{3} \mathrm{TP} *$ and ${ }^{1} \mathrm{TP} * \mathrm{TA}$ response in this spectral range, and thus determine both the triplet cross section and an absolute quantum yield of triplet formation. In concentrated solutions, the population difference between the triplet and singlet are apparent in Fig. 2c, as the intensity of the singlet and triplet infrared excited state absorptions are similar in spite of the two-fold difference in their cross sections. We find that the quantum yield of singlet fission for TIPS-pentacene at $0.075 \mathrm{~mol} / \mathrm{L}$ is near unity, corresponding to a triplet yield of $197 \pm 3 \%$.

Figure $3 \mathrm{~b}$ shows triplet yield measured from TA spectra as a function of TIPSpentacene concentration. At high concentration, the rate of triplet formation reaches an upper limit of $2.2 \times 10^{9} \mathrm{~L} \mathrm{~mol}^{-1} \mathrm{~s}^{-1}$, which is within $30 \%$ of the diffusion limit for a bimolecular reaction in chloroform at room temperature ${ }^{31}$. This result is significant both because it is the maximum observable rate for such a solution, and also because any intermolecular coupling required for singlet fission must occur on a rapid time scale relative to collisions in solution.

Figure $3 \mathrm{~b}$ has an inflection point corresponding to a singlet fission probability of $50 \%$, and the TIPS-pentacene concentration at that point is $7.9 \times 10^{-3} \mathrm{~mol} / \mathrm{L}$. The concentration corresponds to a mean intermolecular separation of $7.4 \mathrm{~nm}$ between TIPS-pentacene molecules. Because singlet fission is limited in this system by the rate at which ${ }^{1} \mathrm{TP}^{*}$ and $\mathrm{TP}$ collide, this distance is thus the average diffusion length of TIPS-pentacene within the singlet lifetime. 


\section{Triplet formation kinetics}

Ultrafast transient absorption spectra of TIPS-pentacene solutions are shown in Fig. 4. In the dilute sample (Fig. $4 \mathrm{a}, 10^{-4} \mathrm{~mol} / \mathrm{L}$ ), the pump pulse excites isolated TIPS-pentacene molecules. These decay on a long time scale consistent with the $13 \mathrm{~ns}$ apparent from Fig. 2a (with fitted functions in Supplementary Fig. S6). In the concentrated sample (Fig. 4b, $0.075 \mathrm{~mol} / \mathrm{L}$ ), the pump pulse still generates TIPS-pentacene singlets, and the initial dynamics are similar to those in Fig. 4a. For instance, both samples exhibit minor spectral changes at early times associated with the solvent dielectric response. By 1000 ps, however, the concentrated sample is dominated by triplet excited state absorption features $(1.28 \mathrm{eV}$ and $1.46 \mathrm{eV}$ ) similar to those in the long time measurements (Fig. 2c). These triplet features grow, and the singlet decays, with a characteristic time of 530 ps (Supplementary Fig. S7). This kinetic is equivalent to a triplet formation rate of $1.7 \times 10^{-6} \mathrm{~mol} \mathrm{~L}^{-1} \mathrm{ps}^{-1}$, with a bimolecular rate constant of $2.2 \times 10^{9} \mathrm{~L} \mathrm{~mol}^{-1} \mathrm{~s}^{-1}$ (see Supplementary Section 7 and Figure S8). This value is within a factor of 2 of the anticipated diffusion limit of TIPSpentacene molecules in chloroform at room temperature, and indicates that the reaction proceeds at its maximum possible rate in solution.

The power-dependence of triplet formation is shown in Supplementary Fig. S9, and after normalising by fluence it is clear that the solution dynamics are linear with incident photon flux. This relationship agrees with the expected result for a sample that exhibits highly efficient singlet fission in the photon-limited kinetic regime, as each incremental increase in light intensity produces a proportionally greater number of triplets. 


\section{Excimer intermediate}

Photoluminescence spectra of TIPS-pentacene (Fig. 5a) show a substantial change in lineshape as the concentration increases. While the dilute spectrum has features that mirror the absorption spectrum and a quantum yield of $75 \%$, at high concentrations, the photoluminescence quantum yield decreases to $<1 \%$, with a featureless spectrum red-shifted by $0.17 \mathrm{eV}$. Time-resolved measurements (Fig. 5b) indicate that the fluorescence lifetime of dilute TIPS-pentacene is $13 \mathrm{~ns}$, and the lifetime of the most concentrated solution is $400 \mathrm{ps}$.

We explored the concentration- and spectral-dependence of this shortened feature. At high concentrations, the shortened lifetime is constant across the lineshape (Supplementary Fig. S10). Although the emission is consistent with an optically allowed transition, the low photoluminescence quantum yield indicates that the excited state is quenched efficiently through a nonradiative pathway. The emission redshift at high concentration does not result from ground state aggregation of $\mathrm{TP}$, as the absorption spectra and DOSY data indicate that the molecules remain dissociated and disordered in the ground state. At intermediate concentrations (Supplementary Fig. S11) however, there is a distribution of different lifetimes, which become progressively shorter as the emission energy decreases. Any self-absorption present in the sample would not produce these effects on the lifetime. Instead, the red-shifted feature arises from a distinct, stabilised interaction of molecules in the excited state, and we attribute the feature to a TIPS-pentacene excimer, ${ }^{1}[\mathrm{TP}-\mathrm{TP}]^{*}$. The observed $400 \mathrm{ps}$ excited state lifetime is a plausible estimate for the singlet fission process and is consistent to within $20 \%$ with the triplet formation rate above (Fig. $4 \mathrm{~b}$ ). Finally, although the lifetime of the emission is nearly constant across the lineshape at $10^{-4} \mathrm{~mol} / \mathrm{L}$ (Supplementary Fig. S12), at low emission energy $(1.46 \mathrm{eV})$ there is evidence for both a fast process (attributed to a small fraction of molecules undergoing singlet fission) and 
a minor slow component. The slight presence of singlet fission in the dilute case further supports our diffusion-limited mechanism.

Our experiments demonstrate singlet fission in TIPS-pentacene solution, which occurs as follows. After photoexcitation, ${ }^{1} \mathrm{TP} *$ and $\mathrm{TP}$ collide, interacting at the maximum rate allowed by diffusion. They form a stabilised species ${ }^{1}[\mathrm{TP}-\mathrm{TP}]^{*}$ that has the energy greater than or equal to the sum of two pentacene triplets ${ }^{32}$, exhibits weak excimer emission and decays rapidly through a single nonradiative pathway on a time scale of 400-530 ps. The excited state population increases after the initial excitation and reaches a maximum of two triplets for every initial singlet.

\section{Computational study of excimer structures}

The observed excimer redshift is a distinct feature of interacting TIPS-pentacene molecules. As a result, this parameter can be used with time-dependent density functional theory (TDDFT) calculations to investigate plausible structures of the ${ }^{1}[\mathrm{TP}-\mathrm{TP}]^{*}$ state that precedes singlet fission. Using a range-separated ${ }^{33}$ functional ( $\omega$ B97X-D), we computed the emission spectral redshift of a dimer of one $\mathrm{TP}$ and one ${ }^{1} \mathrm{TP}^{*}$, relative to ${ }^{1} \mathrm{TP}^{*}$ alone. The starting point is the crystal geometry ${ }^{34}$ shown in Fig. 6 a.

We calculated the emission spectral redshift for varying displacements of $\mathrm{TP}$ and ${ }^{1} \mathrm{TP}^{*}$ at fixed separation of $3.43 \AA$ between planes. The results are shown in Fig. $6 \mathrm{~b}$, and the geometries in red regions show an emission red-shift comparable to experiment. Several test structures from this analysis are shown in Supplementary Fig. S14, with parameters in Supplementary Table S2. We also computed the emission spectral shift for systematic 
variations in the intermolecular separation and relative orientation of the molecules (Supplementary Figs. S15 and S16).

Among the plausible structures from Fig. 6b, the greatest agreement with experiment arises from slip-stacked arrangements of parallel TIPS-pentacene molecules. This slip angle is more pronounced for the excimer than in the crystal, as the crystal geometry (at the origin of Fig. 6b) gives a smaller energetic stabilisation than we observe in solution. Though slipstacked geometries are implicated in J-type coupling between molecules and hypothesised to promote fission in some $\operatorname{cases}^{35,36}$, our experimental results demonstrate that the only geometrical constraint occurs for the excimer, not for molecules in the ground state.

Our results provide some insight into two intermolecular interactions required for singlet fission. First, the initial encounter between ${ }^{1} \mathrm{TP}^{*}$ and $\mathrm{TP}$ in solution is very rapid, and the red-shifted emission from ${ }^{1}[\mathrm{TP}-\mathrm{TP}]^{*}$ demonstrates that they are stabilised. Thus, there is a sufficient driving force for molecules in concentrated solutions to find one another within the singlet lifetime. Second, the long triplet lifetime of TIPS-pentacene in solution (6.5 $\mu \mathrm{s})$ relative to pentacene ${ }^{37}$ or TIPS-pentacene films ${ }^{29}$ suggests that triplet-triplet annihilation is suppressed in solution. Both the first and second processes are hindered in the solid state and in covalent dimers ${ }^{38,39}$ : the reduced freedom of molecules can prevent them from achieving the most favourable geometry for triplet formation, and any triplets that form remain close enough to annihilate. In dilute solutions, the intermolecular distance is too large for diffusing molecules to associate within the radiative lifetime. 


\section{Conclusions}

The results of this study suggest a number of promising directions for future work. While our work supports a bimolecular mechanism for singlet fission in TIPS-pentacene solutions, it also connects to broader mechanistic questions. First, the observed excimer has the energy and multichromophoric character attributed to the state D proposed earlier ${ }^{17}$. The transient association and stabilisation of the TIPS-pentacene excited state energy has similarities with electronic perturbations observed in systems with very different electronic structures, such as polyenes $^{40-43}$. As such, our work suggests that there is scope for universal theories of singlet fission that span many distinct families of chromophores ${ }^{4,44,45}$.

Because we identified material design principles and a mechanistic pathway for understanding singlet fission, these findings will inspire the synthesis of the next generation of light-harvesting materials and allow the harvesting of energy that is typically lost to relaxation $^{46}$. Beyond the facile processibility enabled by the use of a soluble material ${ }^{47}$, the $\sim 200 \%$ triplet yield in solution also suggests the use of TIPS-pentacene in a liquid photocatalytic cell based on singlet fission.

In conclusion, we have demonstrated the complete fission of singlet excitons into two triplet excitons in solution. We have observed the distinct signature of an excimer intermediate to singlet fission, and our results lead to a molecular mechanism. From these results, we anticipate new singlet fission devices, new synthesis to promote intermolecular interactions, and new ultrafast studies on the interactions of disordered chromophores. 


\section{$\underline{\text { References }}$}

1. Singh, S., Jones, W. J., Siebrand, W., Stoicheff, B. P. \& Schneider, W. G. Laser Generation of Excitons and Fluorescence in Anthracene Crystals. The Journal of Chemical Physics 42, 330 (1965).

2. Swenberg, C. E. \& Tracy, W. T. Bimolecular radiationless transitions in crystalline tetracene. Chemical Physics Letters 2, 327-328 (1968).

3. Geacintov, N., Pope, M. \& Vogel, F. Effect of Magnetic Field on the Fluorescence of Tetracene Crystals: Exciton Fission. Physical Review Letters 22, 593-596 (1969).

4. Smith, M. B. \& Michl, J. Singlet Fission. Chemical Reviews 110, 6891-936 (2010).

5. Smith, M. B. \& Michl, J. Recent advances in singlet fission. Annual review of Physical Chemistry 64, 361-86 (2013).

6. Merrifield, R. E. Diffusion and mutual annihilation of triplet excitons in organic crystals. Accounts of Chemical Research 1, 129-135 (1968).

7. Merrifield, R. E., Avakian, P. \& Groff, R. P. Fission of Singlet Excitons into Pairs of Triplet Excitons in Tetracene Crystals. Chemical Physics Letters 3, 155-157 (1969).

8. Chan, W., Ligges, M. \& Zhu, X.-Y. The energy barrier in singlet fission can be overcome through coherent coupling and entropic gain. Nature Chemistry 4, 840-5 (2012).

9. Jundt, C. et al. Exciton dynamics in pentacene thin films studied by pump-probe spectroscopy. Chemical Physics Letters 241, 84-88 (1995).

10. Vilar, M. R., Heyman, M. \& Schott, M. Spectroscopy of Low-Energy Electrons Backscattered from an Organic Solid Surface: Pentacene. Chemical Physics Letters 94, 522-6 (1983).

11. Shockley, W. \& Queisser, H. J. Detailed Balance Limit of Efficiency of p-n Junction Solar Cells. Journal of Applied Physics 32, 510 (1961).

12. Hanna, M. C. \& Nozik, A. J. Solar conversion efficiency of photovoltaic and photoelectrolysis cells with carrier multiplication absorbers. Journal of Applied Physics 100, 074510 (2006).

13. Jadhav, P. J. et al. Triplet exciton dissociation in singlet exciton fission photovoltaics. Advanced Materials 24, 6169-74 (2012).

14. Ehrler, B., Wilson, M. W. B., Rao, A., Friend, R. H. \& Greenham, N. C. Singlet exciton fission-sensitized infrared quantum dot solar cells. Nano Letters 12, 1053-7 (2012). 
15. Paci, I. et al. Singlet fission for dye-sensitized solar cells: can a suitable sensitizer be found? Journal of the American Chemical Society 128, 16546-53 (2006).

16. Zimmerman, P. M., Zhang, Z. \& Musgrave, C. B. Singlet fission in pentacene through multi-exciton quantum states. Nature Chemistry 2, 648-52 (2010).

17. Zimmerman, P. M., Bell, F., Casanova, D. \& Head-Gordon, M. Mechanism for singlet fission in pentacene and tetracene: from single exciton to two triplets. Journal of the American Chemical Society 133, 19944-52 (2011).

18. Greyson, E. C., Vura-Weis, J., Michl, J. \& Ratner, M. A. Maximizing singlet fission in organic dimers: theoretical investigation of triplet yield in the regime of localized excitation and fast coherent electron transfer. The Journal of Physical Chemistry $B$ 114, 14168-77 (2010).

19. Chan, W.-L. et al. Observing the multiexciton state in singlet fission and ensuing ultrafast multielectron transfer. Science (New York, N.Y.) 334, 1541-5 (2011).

20. Zimmerman, P. M., Musgrave, C. B. \& Head-Gordon, M. A Correlated Electron View of Singlet Fission. Accounts of Chemical Research 46, 1339-1347 (2013).

21. Beljonne, D., Yamagata, H., Brédas, J. L., Spano, F. C. \& Olivier, Y. Charge-Transfer Excitations Steer the Davydov Splitting and Mediate Singlet Exciton Fission in Pentacene. Physical Review Letters 110, 226402 (2013).

22. Roberts, S. T. et al. Efficient singlet fission discovered in a disordered acene film. Journal of the American Chemical Society 134, 6388-400 (2012).

23. Burdett, J. J., Müller, A. M., Gosztola, D. \& Bardeen, C. J. Excited state dynamics in solid and monomeric tetracene: The roles of superradiance and exciton fission. The Journal of Chemical Physics 133, 144506 (2010).

24. Burdett, J. J., Gosztola, D. \& Bardeen, C. J. The dependence of singlet exciton relaxation on excitation density and temperature in polycrystalline tetracene thin films: kinetic evidence for a dark intermediate state and implications for singlet fission. The Journal of Chemical Physics 135, 214508 (2011).

25. Sheraw, C. D., Jackson, T. N., Eaton, D. L. \& Anthony, J. E. Functionalized Pentacene Active Layer Organic Thin-Film Transistors. Advanced Materials 15, 2009-2011 (2003).

26. Giri, G. et al. Tuning charge transport in solution-sheared organic semiconductors using lattice strain. Nature 480, 504-8 (2011).

27. Gundlach, D. J. et al. Contact-induced crystallinity for high-performance soluble acene-based transistors and circuits. Nature Materials 7, 216-21 (2008).

28. Wilson, M. W. B. et al. Ultrafast dynamics of exciton fission in polycrystalline pentacene. Journal of the American Chemical Society 133, 11830-3 (2011). 
29. Ramanan, C., Smeigh, A. L., Anthony, J. E., Marks, T. J. \& Wasielewski, M. R. Competition between singlet fission and charge separation in solution-processed blend films of 6,13-bis(triisopropylsilylethynyl)pentacene with sterically-encumbered perylene-3,4:9,10-bis(dicarboximide)s. Journal of the American Chemical Society 134, 386-97 (2012).

30. Van Hal, P. A. et al. Photoinduced singlet and triplet energy transfer in fullereneoligothiophene-fullerene triads. Synthetic Metals 116, 123-127 (2001).

31. Berg, O. G. \& von Hippel, P. H. Diffusion-Controlled Macromolecular Interactions. Ann. Rev. Biophys. Biophys. Chem 14, 131-60 (1985).

32. Ehrler, B. et al. In situ measurement of exciton energy in hybrid singlet-fission solar cells. Nature Communications 3, 1019 (2012).

33. Dreuw, A., Weisman, J. L. \& Head-Gordon, M. Long-range charge-transfer excited states in time-dependent density functional theory require non-local exchange. The Journal of Chemical Physics 119, 2943 (2003).

34. Mannsfeld, S. C. B., Tang, M. L. \& Bao, Z. Thin film structure of triisopropylsilylethynyl-functionalized pentacene and tetraceno[2,3-b]thiophene from grazing incidence X-ray diffraction. Advanced Materials 23, 127-31 (2011).

35. Johnson, J. C., Nozik, A. J. \& Michl, J. The Role of Chromophore Coupling in Singlet Fission. Accounts of Chemical Research 46, 1290-9 (2013).

36. Renaud, N., Sherratt, P. A. \& Ratner, M. A. Mapping the Relation between Stacking Geometries and Singlet Fission Yield in a Class of Organic Crystals. The Journal of Physical Chemistry Letters 4, 1065-1069 (2013).

37. Rao, A. et al. Exciton fission and charge generation via triplet excitons in pentacene/C60 bilayers. Journal of the American Chemical Society 132, 12698-703 (2010).

38. Müller, A. M., Avlasevich, Y. S., Müllen, K. \& Bardeen, C. J. Evidence for exciton fission and fusion in a covalently linked tetracene dimer. Chemical Physics Letters 421, 518-522 (2006).

39. Müller, A. M., Avlasevich, Y. S., Schoeller, W. W., Müllen, K. \& Bardeen, C. J. Exciton fission and fusion in bis(tetracene) molecules with different covalent linker structures. Journal of the American Chemical Society 129, 14240-50 (2007).

40. Wang, C. \& Tauber, M. J. High-yield singlet fission in a zeaxanthin aggregate observed by picosecond resonance Raman spectroscopy. Journal of the American Chemical Society 132, 13988-91 (2010).

41. Schulten, K., Ohmine, I. \& Karplus, M. Correlation effects in the spectra of polyenes. The Journal of Chemical Physics 64, 4422 (1976). 
42. Gradinaru, C. C. et al. An unusual pathway of excitation energy deactivation in carotenoids: singlet-to-triplet conversion on an ultrafast timescale in a photosynthetic antenna. Proceedings of the National Academy of Sciences of the United States of America 98, 2364-9 (2001).

43. Barford, W. Electronic and Optical Properties of Conjugated Polymers. (Oxford University Press, 2005).

44. Berkelbach, T. C., Hybertsen, M. S. \& Reichman, D. R. Microscopic theory of singlet exciton fission. I. General formulation. The Journal of Chemical Physics 138, 114102 (2013).

45. Ito, S., Minami, T. \& Nakano, M. Diradical Character Based Design for Singlet Fission of Condensed- Ring Systems with $4 n \pi$ Electrons. The Journal of Physical Chemistry C 116, 19729-36 (2012).

46. Chan, W.-L., Tritsch, J. R. \& Zhu, X.-Y. Harvesting singlet fission for solar energy conversion: one- versus two-electron transfer from the quantum mechanical superposition. Journal of the American Chemical Society 134, 18295-302 (2012).

47. Azarova, N. A. et al. Fabrication of organic thin-film transistors by spray-deposition for low-cost, large-area electronics. Organic Electronics 11, 1960-1965 (2010).

\section{Acknowledgements}

B.J.W. was supported by a Herchel Smith Research Fellowship. A.J.M. received funding from a Marie Curie Scholarship. D.B. is a FNRS Research Director. Both A.J.M and D.B. acknowledge support from the European Community's Initial Training Network SUPERIOR (PITN-GA-2009-238177). Further funding for this project came from the Engineering and Physical Sciences Research Council (EPSRC) and a pump-prime grant from the Winton Programme for the Physics of Sustainability. We thank Akshay Rao, Kerr Johnson and Simon Gélinas for general discussion, and Duncan Howe for assistance with diffusionordered NMR spectroscopy. 


\section{$\underline{\text { Author Contributions }}$}

B.J.W. conceived of the project, B.J.W. and A.J.M. planned and carried out experiments, A.J.M. performed calculations, B.J.W., A.J.M, D.B. and R.H.F. discussed experiments and results, and B.J.W., A.J.M. and R.H.F. wrote the manuscript.

Competing Interests The authors declare no competing financial interests associated with this work.

Correspondence Correspondence should be addressed to R.H.F. (email: rhf10@cam.ac.uk). 
Figure 1 Overview of TIPS-pentacene. a, Scheme for the mechanism of singlet fission in TIPS-pentacene solution. One excited singlet molecule and one ground state molecule associate in the rate limiting step to form an excimer. This intermediate decays to two triplets. Blue arrows are reactions, red arrows are optical transitions. b, Structure of TIPS-pentacene. c, Ground-state absorption spectra of TIPS-pentacene dissolved in chloroform and as a spuncast film. Solid state TIPS-pentacene has an aggregate feature at $1.75 \mathrm{eV}$.

Figure 2 Transient absorption spectra of TIPS-pentacene. a, TIPS-pentacene solution at $10^{-4} \mathrm{~mol} / \mathrm{L}$. The dynamics are dominated by singlets and decay within $13 \mathrm{~ns}$ after peak of the excitation pulse (at $2.6 \mathrm{~ns}$ ). b, Fixed-time spectrum of the TIPS-pentacene singlet excited state from (a) and of the triplet excited state from sensitisation experiments. The powerindependent excited state molar absorptivity was measured for the singlet, and the triplet was normalised to the singlet at 1.9-2.0 eV. c, TIPS-pentacene solution, $0.075 \mathrm{~mol} / \mathrm{L}$. In addition to singlet features there are long-lived signals due to triplets. The ground state bleach at $1.9 \mathrm{eV}$ has both a delayed increase and a decay that is attributable entirely to triplets, which constitutes direct evidence for complete singlet fission.

Figure 3 Triplet yield in TIPS-pentacene solutions. a, Population dynamics of TIPSpentacene in solution, extracted from Fig. $2 \mathrm{c}$ at a solution concentration of $0.075 \mathrm{~mol} / \mathrm{L}$. Kinetics were sampled at 1.27-1.29 eV for triplets and 0.88-0.95 eV for singlets. The triplet population is referenced to the singlet through the excited state absorption coefficients from 1.9-2.0 eV. Decay of the singlet population is matched by the triplet rise, and the triplet yield is $200 \%$. b, Plot of triplet yield as a function of concentration. The quantum yield approaches unity at high concentration, resulting in a triplet yield of $\sim 200 \%$. Error bars reflect 
measurements on multiple samples, and the mean intermolecular distance is shown for reference.

Figure 4 Ultrafast transient absorption spectra of TIPS-pentacene with a time resolution of 0.28 ps. Black lines at 1 ps indicate a change from linear to logarithmic timescale. a, TIPSpentacene solution at $10^{-4} \mathrm{~mol} / \mathrm{L}$. The spectrum is dominated by the singlet excited state absorption, which rises within the instrument response and exhibits solvent relaxation at early times. b, TIPS-pentacene solution at $0.075 \mathrm{~mol} / \mathrm{L}$. The initial excited population is again composed of singlets. Substantial triplet growth occurs from 100 ps as indicated by the absorptions at $1.27-1.29 \mathrm{eV}$ and $1.44-1.47 \mathrm{eV}$, and is accompanied by accelerated singlet decay $(1.30-1.42 \mathrm{eV})$.

Figure 5 Photoluminescence of TIPS-pentacene solutions. a, Plot of the emission spectra as a function of concentration, normalised at $1.7 \mathrm{eV}$. The excimer emission at $1.7 \mathrm{eV}$ becomes relatively more prominent at high concentrations. $\mathbf{b}$, Time-resolved emission of dilute and concentrated TIPS-pentacene. Single-exponential fits give a lifetime of $13 \mathrm{~ns}$ for the dilute sample and $400 \mathrm{ps}$ for the concentrated sample. Coloured bars in a indicate emission energies for each sample.

Figure 6 Time-dependent density functional theory analysis of TIPS-pentacene excimer structures. a, Starting geometry for the TIPS-pentacene excimer from the solid-state crystal structure. Trasverse (T) and longitudinal (L) axes are indicated. b, Effect of $\pi$-stacking on the emission spectral redshift between the excimer and monomer structures, for parallel 
molecular planes separated by $3.43 \AA$. The crystal geometry is the origin. An emission redshift of $170 \mathrm{meV}$ is observed, and regions coloured red are comparable to experiment. 

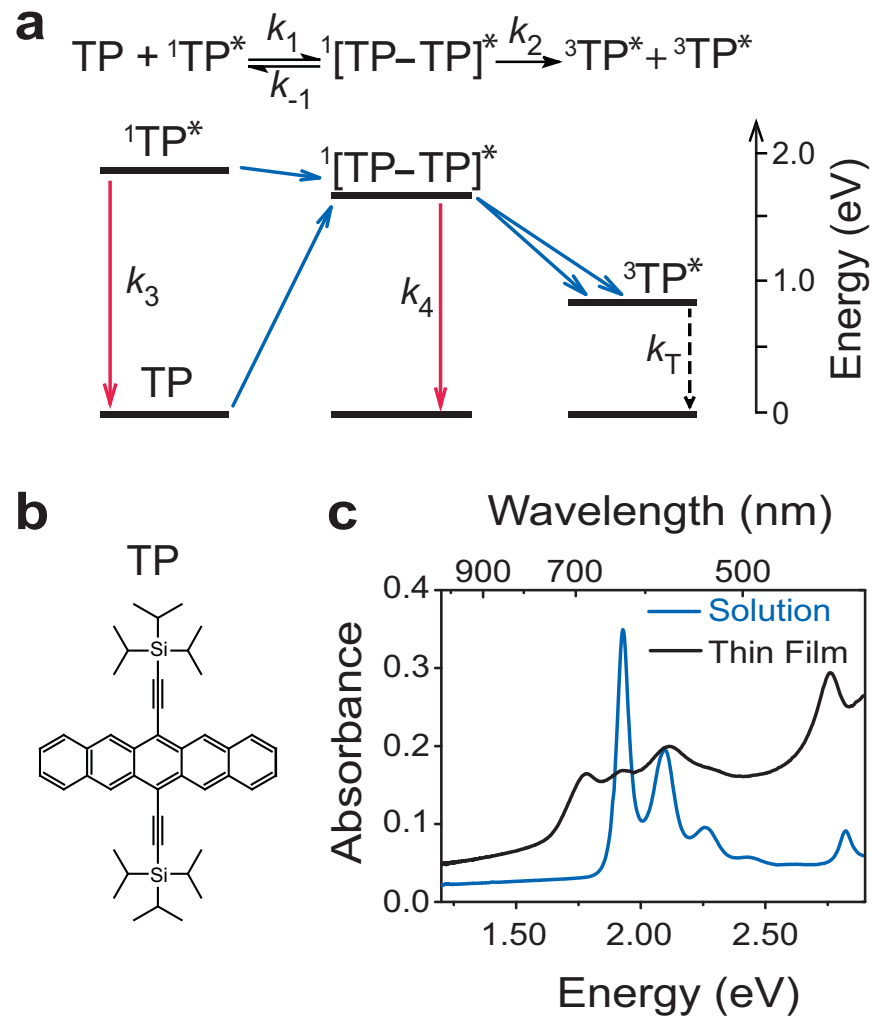

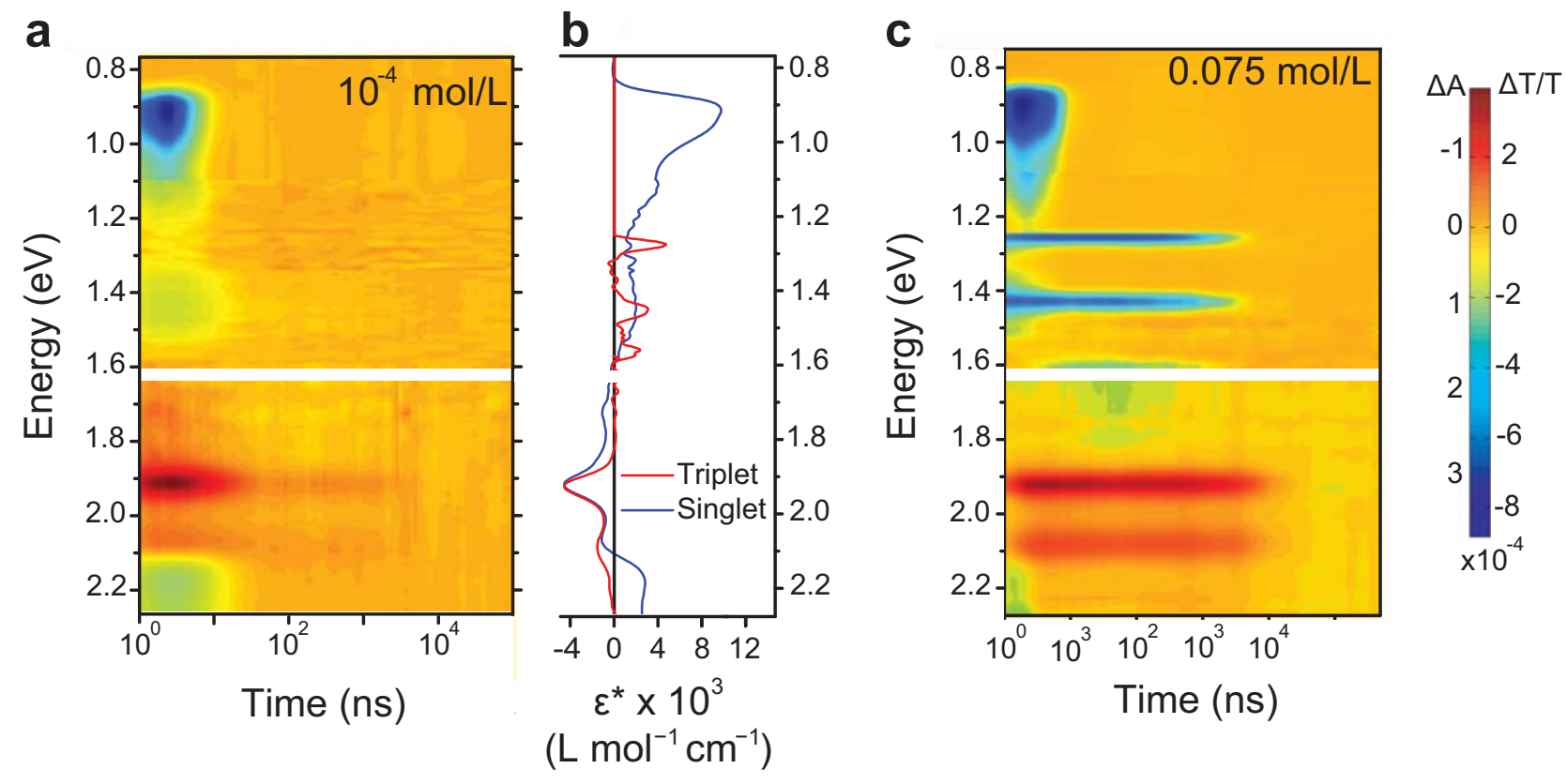

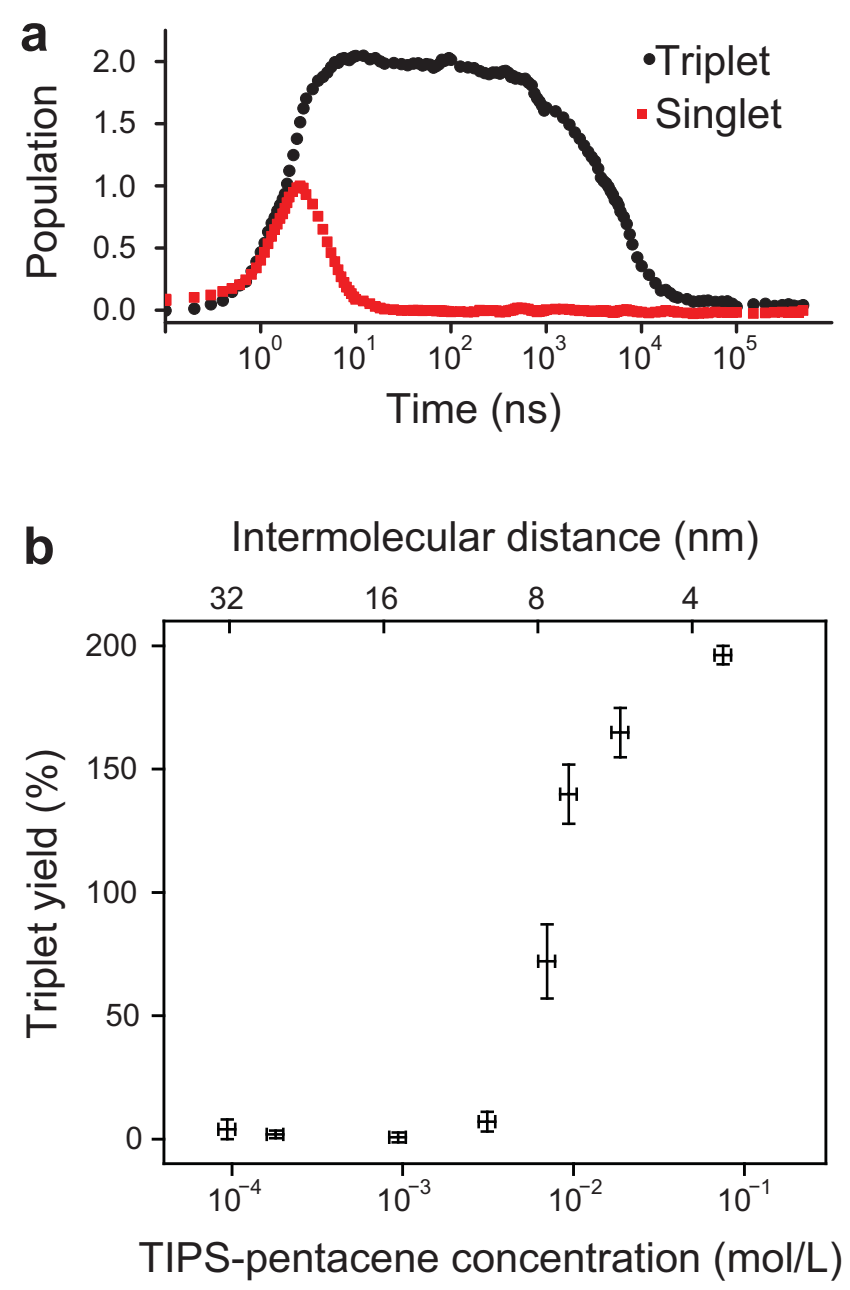

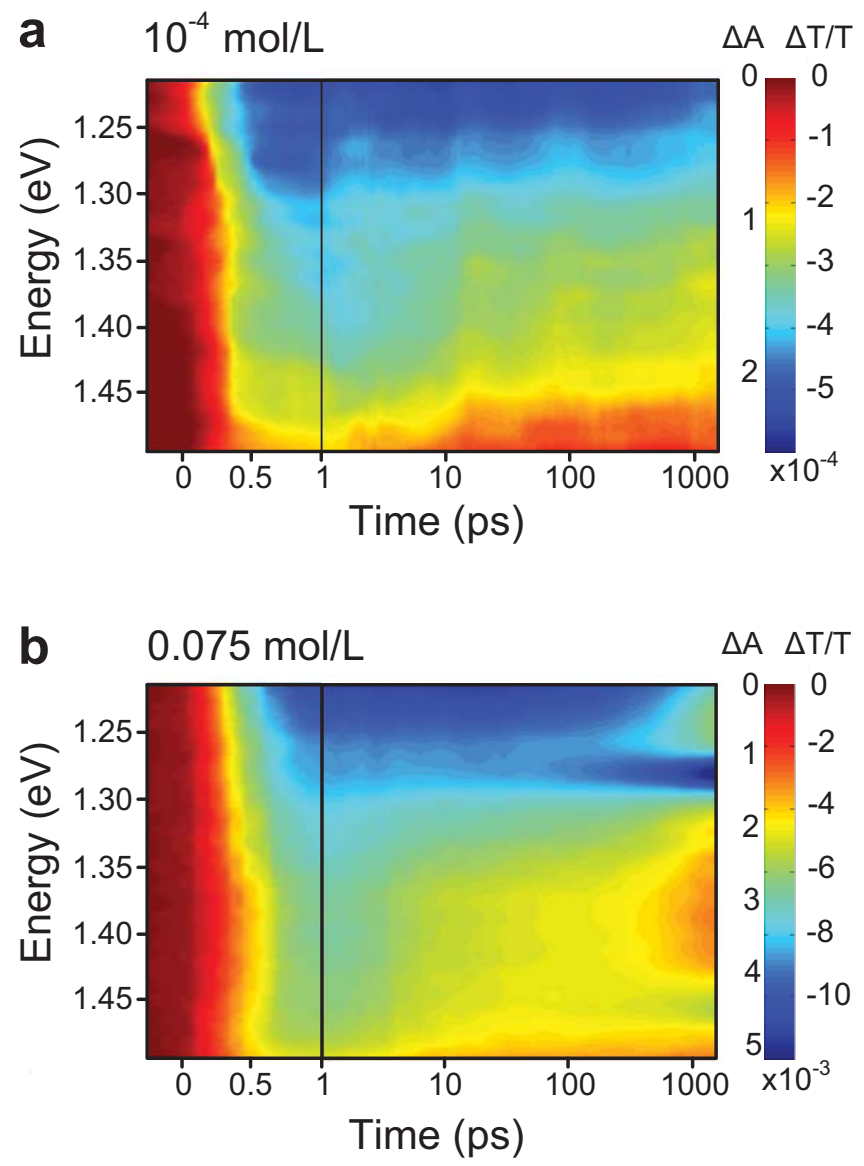

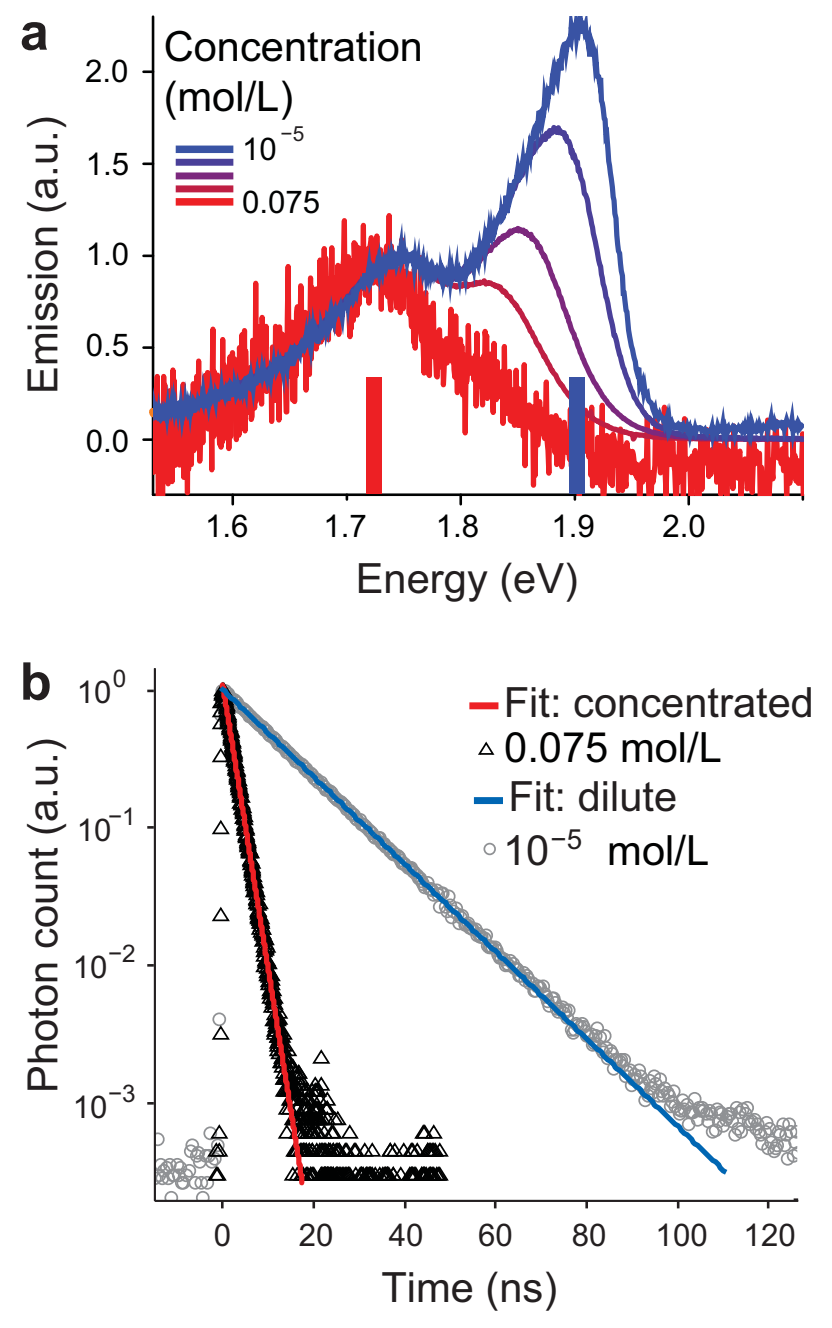

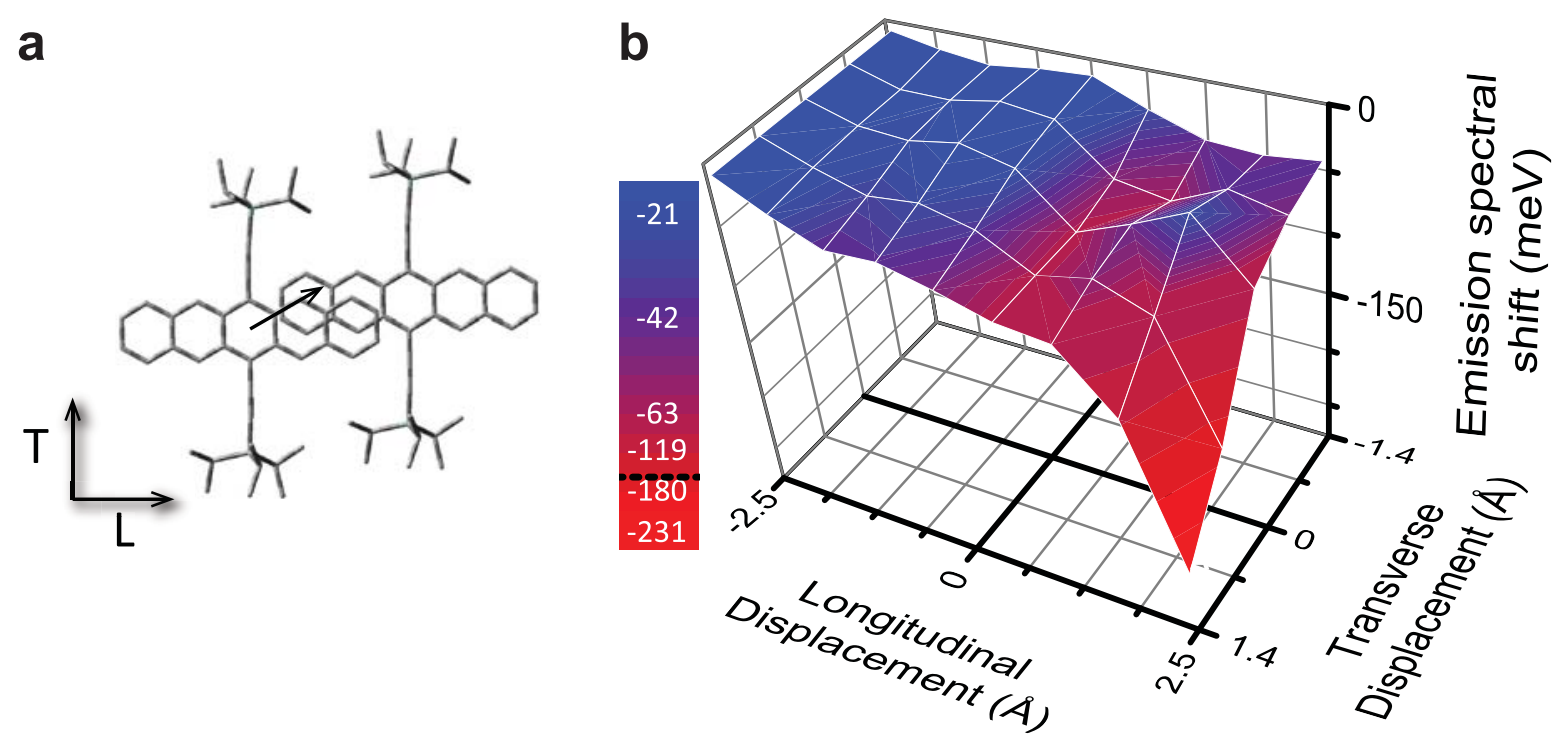


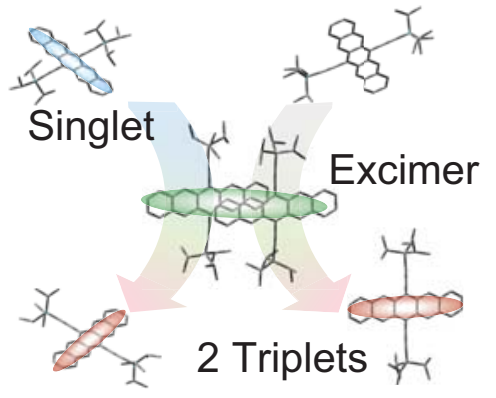


Singlet exciton fission produces two triplet excited states from one excited singlet, through interchromophoric coupling which is thought to require local order. Here, we report $200 \%$ triplet yield and diffusion-limited triplet formation in solutions of TIPS-pentacene. Kinetic studies reveal an excimer intermediate and suggest design principles to promote singlet fission. 